Bundesgesundheitsbl 2014 $\cdot 57: 733-743$

DOI 10.1007/s00103-014-1972-x

c) Springer-Verlag Berlin Heidelberg 2014
Bekanntmachung des Umweltbundesamtes

\section{Richtwerte für 1-Butanol in der Innenraumluft}

\section{Stoffidentifizierung [1]}

$\begin{array}{ll}\begin{array}{l}\text { Systematischer } \\ \text { Name: }\end{array} & \begin{array}{l}\text { 1-Butanol } \\ \text { nynonyme: }\end{array} \\ & \begin{array}{l}\text { alkohol, n-Butan-1-ol, } \\ \text { 1-Hydroxybutan }\end{array} \\ \text { CLP-Index-Nr: } & 603-004-00-6 \\ \text { EG-Nr: } & 200-751-6 \\ \text { CAS-Nr.: } & 71-36-3 \\ \text { Summenformel: } & \mathrm{C}_{4} \mathrm{H}_{10} \mathrm{O} \\ \text { Strukturformel: } & \mathrm{H}_{3} \mathrm{C}\end{array}$

\subsection{Physikalische und chemische} Eigenschaften [1]

Molekulargewicht: 74,12 g/mol

Schmelzpunkt: $-89,9^{\circ} \mathrm{C}$

Siedepunkt: $117,6^{\circ} \mathrm{C}$ bei $1013 \mathrm{hPa}$

Dichte: $0,81 \mathrm{~g} / \mathrm{cm}^{3}$ bei $20^{\circ} \mathrm{C}$

Dampfdruck: $0,56 \mathrm{kPa}$ bei $20^{\circ} \mathrm{C}$

Relative Gasdichte $(\mathrm{Luft}=1): 2,5$

Wasserlöslichkeit: $77 \mathrm{~g} / \mathrm{l}$ bei $20^{\circ} \mathrm{C}$

Verteilungskoeffizient $\lg \mathrm{K}_{\text {Octanol/Wasser: }}$ 0,88

Umrechnung (bei $20^{\circ} \mathrm{C}$ ): $1 \mathrm{mg} / \mathrm{m}^{3}$ $=0,33 \mathrm{ppm}, 1 \mathrm{ppm}=3,08 \mathrm{mg} / \mathrm{m}^{3}$

\subsection{Stoffeigenschaften und Anwendung}

1-Butanol ist eine farblose, brennbare, in Wasser gut lösliche Flüssigkeit mit einem charakteristischen Geruch, der als „weinartig“ beschrieben wird [2], aber auch als „herb fuselähnlich mit Bananenaroma“ sowie als „süßlich ranzig“ [3]. Die Ge- ruchsqualität wird als „,neutral bis leicht unangenehm" angesehen [4].

1-Butanol kommt von Natur aus in vielen Nahrungsmitteln vor und entsteht beim mikrobiologischen Abbau von Kohlenhydraten. Die großtechnische Herstellung aus erneuerbaren Rohstoffen, insbesondere Polysacchariden, aber auch aus Propantriol als Abfallprodukt der Synthese von Fettsäuremethylestern aus Triglyceriden, mithilfe von Mikroorganismen wird derzeit intensiv diskutiert. Auf diese Weise hergestelltes „Biobutanol“ soll statt Ethanol als Treibstoffzusatz Verwendung finden [5]. Die Hauptmenge an 1-Butanol wird jedoch gegenwärtig großtechnisch durch Hydroformylierung von Propen, Kohlenstoffmonoxid und Wasserstoff zu Butanal und nachfolgende Reduktion des Aldehyds mit Wasserstoff hergestellt [1, 2]. Verwendung findet 1-Butanol in erster Linie als Ausgangsstoff zur Herstellung von Lösemitteln wie etwa Butylacetat und Glykolbutylethern und -estern sowie von Phthalaten und Acrylaten. Außerdem dient 1-Butanol selbst als Lösemittel, vor allem in Farben, Lacken, Harzen und Farbentfernern sowie manchen Kosmetika und wird in der Oberflächenveredelung von Kleidung eingesetzt [1].

\section{Exposition}

\subsection{Innenraumluft}

Zum Vorkommen von 1-Butanol in der Luft von Wohnungen, Schulen und Kin- dergärten in Deutschland liegen einige Angaben vor (• Tab. 1). Demnach liegen die Konzentrationen im Median im Bereich um $10 \mu \mathrm{g} / \mathrm{m}^{3}$, bei Spitzenwerten (95. Perzentil) bis $200 \mu \mathrm{g} / \mathrm{m}^{3}$. Nach Angaben von Hoffmann und Plieninger [6] zählt 1-Butanol zu den Substanzen, die in etwa $90 \%$ der Räume gefunden wurden. Dies deckt sich mit den Angaben von Eis et al. [7], denen zufolge 1-Butanol die dominierende Verbindung unter den einwertigen Alkoholen darstellt, die bei über $85 \%$ der Proben in der Innenraumluft bestimmt wurde. Ein klarer zeitlicher Trend lässt sich aus den Daten nicht ableiten.

\subsection{Nahrungsmittel und Verbraucherprodukte}

1-Butanol entsteht im Zuge des Abbaus von Kohlenhydraten bei der Herstellung alkoholischer Getränke. Nachgewiesen wurde dieser Alkohol außerdem in Früchten wie Melonen und Äpfeln sowie verschiedenen Nahrungsmitteln wie Käse, erhitzter Milch und gekochtem Reis sowie bei der Popcornbereitung, aber auch in der Muttermilch [1, 3]. Ältere quantitative Angaben aus den 1960er-Jahren über den Höchstgehalt von 1-Butanol in Lebensmitteln belaufen sich auf Werte von $12 \mathrm{mg} / \mathrm{l}$ in Getränken, $7 \mathrm{mg} / \mathrm{kg}$ in Speiseeis und $32 \mathrm{mg} / \mathrm{kg}$ in Backwaren [8].

Neben der oralen Exposition über Nahrungs- und Genussmittel kann eine dermale und inhalative Exposition gegenüber 1-Butanol aus Verbraucherpro- 


\begin{tabular}{|c|c|c|c|c|c|c|c|}
\hline Innenraum/Studie & $\mathrm{N}$ & $B G\left(\mu g / m^{3}\right)$ & $\begin{array}{l}N>B G \\
(\%>B G)\end{array}$ & $\begin{array}{l}\text { Median } \\
\left(\mu \mathrm{g} / \mathrm{m}^{3}\right)\end{array}$ & $\begin{array}{l}\text { 95. Perzentil } \\
\left(\mu \mathrm{g} / \mathrm{m}^{3}\right)\end{array}$ & $\begin{array}{l}\text { Maximalwert } \\
\left(\mu \mathrm{g} / \mathrm{m}^{3}\right)\end{array}$ & Referenz \\
\hline Umwelt-Survey 1985/86 & 479 & n.a. & n. a. & 0,7 & 4,1 & 19 & [9] \\
\hline Erwachsenen-Umwelt-Survey 1990-1992 & 113 & n.a. & $53(44,5 \%)$ & $<1$ & 31 & 221 & [10] \\
\hline n. a./Berlin 1994-1999 & 23 & n. a. & n. a. & 27 & 200 & n. a. & [7] \\
\hline n. a./Bayern 1995-2000 & 382 & n.a. & n.a. & 16 & 120 & n. a. & [7] \\
\hline Wohn- und Büroräume/Berlin 1999-2003 & 50 & 2 & $47(94 \%)$ & 12,5 & 53,5 & 169 & [7] \\
\hline $\begin{array}{l}\text { Vor allem Schlaf-, Wohnzimmer, Büroräu- } \\
\text { me, Klassenräume } 2008\end{array}$ & 2284 & $2,2(0,5-8)$ & $2040(89,3 \%)$ & 11,0 & 73,9 & 3422 & [6] \\
\hline Wohnungen, S-H, 2000-2001 & 72 & 0,85 & n.a. & 7,3 & n. a. & 41 & [11] \\
\hline Wohnungen, KUS 2003-2006 & 555 & 1 & $544(98 \%)$ & 5,4 & 17,6 & 71,6 & [12] \\
\hline $\begin{array}{l}\text { Schulen und Kindergärten, S-H, 2005- } \\
2007\end{array}$ & 285 & 2 & $(93 \%)$ & 3,0 & 12,0 & 39 & [13] \\
\hline Schulen 2004-2009 & 387 & 19 & $219(57)$ & n. a. & 39 & n. a. & [14] \\
\hline Büroräume, 2001-2005 & 898 & 10 & $526(59)$ & n. a. & 50 & n. a. & [15] \\
\hline Büroräume, 2006-2010 & 975 & 10 & $666(68)$ & n. a. & 58 & n. a. & [15] \\
\hline
\end{tabular}

n. a. nicht angegeben

dukten wie Kosmetika, Farben und Lacken erfolgen [1].

\subsection{Gesamtexposition}

Aufgrund des natürlichen Vorkommens von 1-Butanol in Nahrungsmitteln ist von einer ubiquitären Belastung auszugehen. Zur Höhe der Exposition über die einzelnen Aufnahmepfade und der Gesamtexposition liegen jedoch keine quantitativen Angaben vor.

\section{Toxikokinetik}

Zur Simulation der Kinetik von 1-Butylacetat, 1-Butanol, Butanal und Butansäure wurde ein pharmakokinetisches Modell entwickelt, das im Rahmen der Bewertung der Toxizität von 1-Butanol durch die US-amerikanische Umweltbehörde zur Extrapolation tierexperimenteller Befunde auf den Menschen herangezogen wurde $[2,16]$.

\subsection{Aufnahme und Verteilung}

Die inhalative pulmonale Retentionsrate von 1-Butanol lag in Untersuchungen an 12 Probanden in Ruhe bei $47 \%$ und sank bei leichter körperlicher Belastung (50$150 \mathrm{~W}$ ) auf etwa $40 \%$ ab. Dabei war die prozentuale Aufnahme im untersuchten Konzentrationsbereich (300 bzw. 600 mg/ $\mathrm{m}^{3}$ ) unabhängig von der Konzentration [17]. In einer anderen Untersuchung lag diese Retentionsrate bei Inhalation von $200 \mathrm{mg} / \mathrm{m}^{3}$ unabhängig von der körperlichen Belastung (bis $75 \mathrm{~W}$ ) bei $60 \%$ [18]. Ein ähnlicher Wert von $55 \%$ wurde tierexperimentell auch bei Hunden nach bis zu 6-stündiger Exposition gegenüber $150 \mathrm{mg} / \mathrm{m}^{3}$ ermittelt [2].

Bei oraler Verabreichung wird 1-Butanol praktisch vollständig resorbiert. Ratten, denen 4,5, 45 bzw. $450 \mathrm{mg}\left[1^{14} \mathrm{C}\right]-1$ Butanol/kg KG in Maisöl gelöst verabreicht wurden, schieden binnen $24 \mathrm{~h}$ unabhängig von der verabreichten Dosis nur $1 \%$ der verabreichten Dosis mit den Faeces wieder aus [19]. Über die dermale Aufnahme von gasförmigem 1-Butanol liegen keine Angaben vor. Nach Abschätzungen auf Basis von In-vitro-Untersuchungen mit isolierter menschlicher Haut ist die Aufnahme von flüssigem 1-Butanol durch die Haut gering (1\%).

Ins Blut aufgenommenes 1-Butanol wird im Körper sehr schnell verteilt und metabolisiert. Bei inhalativer 30-minütiger Exposition von Probanden gegenüber $150 \mathrm{mg} / \mathrm{m}^{3}$ war im Blut kein 1-Butanol nachweisbar (Nachweisgrenze $80 \mu \mathrm{g} / \mathrm{l}$ ). Wurde die Konzentration auf $600 \mathrm{mg} / \mathrm{m}^{3}$ erhöht und die absolute Aufnahme durch vermehrte Respiration bei körperlicher Aktivität erhöht, so wurden maximale Blutspiegel von 1,3 mg/l nachgewiesen, die binnen 30 min nach Ende der Exposition wieder unter die Nachweisgrenze fielen [18]. Im Tierversuch war nach oraler Verabreichung von $450 \mathrm{mg}\left[1^{14} \mathrm{C}\right]-1-\mathrm{Bu}-$ tanol/kg KG in Maiskeimöl an fastende Ratten die Spitzenkonzentration im Blutplasma mit 70,9 $\mu \mathrm{g} / \mathrm{ml}$ binnen $1 \mathrm{~h}$ erreicht, gefolgt von einem raschen Abfall unter $10 \mu \mathrm{g} / \mathrm{ml}$ nach $2 \mathrm{~h}$. Vier Stunden nach Verabreichung war kein Butanol im Plasma mehr nachweisbar [19]. In vitro bestimmte Gewebe:Blut-Verteilungskoeffizienten von 0,78-1,08 für Muskel-, Hirn- Nieren- und Fettgewebe weisen darauf hin, dass sich 1-Butanol gleichmäßig in alle Organe verteilt. In-vivo-Untersuchungen mit radioaktiv markiertem 1-Butanol an Ratten und Menschen bestätigen dies und belegen darüber hinaus, dass die Blut-Hirn-Schranke rasch und in hohem Maße durchdrungen wird $[2,18]$. Nach oraler Verabreichung von $\left[1{ }^{14} \mathrm{C}\right]-1-B u t a n o l$ traten die höchsten ${ }^{14} \mathrm{C}$-Aktivitäten $4-8 \mathrm{~h}$ später auf, wobei nach 4 h 0,24\% der Aktivität in der Niere und nach 8 h 3,88\% in der Leber gefunden wurden, mit 0,04\% war der Anteil im Gehirn gering. Etwa $15 \%$ der eingesetzten ${ }^{14} \mathrm{C}$-Aktivität waren nach $24 \mathrm{~h}$ noch im Körper verblieben [19]. Da lediglich die ${ }^{14} \mathrm{C}$-Aktivität gemessen wurde, nicht aber die Konzentration des Ausgangsstoffs oder an Metaboliten, liefern diese Befunde nur Anhaltspunkte für Höchstgehalte an 1-Butanol. Der tatsächliche Gehalt ist wegen der raschen Metabolisierung erheblich niedriger, wurde jedoch nicht bestimmt. 


\subsection{Metabolismus und Ausscheidung}

Die Metabolisierung von 1-Butanol erfolgt tierexperimentellen und In-vitroDaten zufolge in erster Linie durch Oxidation mittels Alkoholdehydrogenase $(\mathrm{ADH})$ in der Leber, in geringerem Maße auch durch hepatische Cytochrom-P450Monoxygenasen, deren Aktivität durch Ethanol induzierbar ist. Eine Glucuronidierung oder Sulfatierung von unverändertem 1-Butanol erfolgt nur in untergeordnetem Maß. In-vitro-Untersuchungen mit Zytosolpräparaten aus Rattenleber- bzw. -lungenzellen lassen nur eine geringe Aktivität der $\mathrm{ADH}$ in der Lunge erkennen. Dies wird als Hinweis darauf gesehen, dass der Lunge kein nennenswerter Anteil am Abbau von 1-Butanol über diesen Stoffwechsel zukommt [2].

Der im ersten Oxidationsschritt gebildete Metabolit Butanal wird rasch durch Aldehyddehydrogenasen weiter zu Butansäure oxidiert, die als kurzkettige Fettsäure im Intermediärstoffwechsel verwertet und letztendlich zu Kohlenstoffdioxid und Wasser oxidiert wird. Vergleichende In-vitro-Untersuchungen zum $\mathrm{ADH}-\mathrm{ka}$ talysierten Abbau von Alkoholen ergaben, dass 1-Butanol rascher als andere Alkohole und etwa 1,5-fach schneller als Ethanol oxidiert wird. Der Abbau von 1-Butanol in der Leber wird durch Ethanol kompetitiv gehemmt [2].

Die Ausscheidung von 1-Butanol und seinen Metaboliten erfolgt nach Befunden an Ratten überwiegend über die Atemwege. Nach oraler Verabreichung von $\left[1^{14} \mathrm{C}\right]$-markiertem 1-Butanol (s. oben) wurden binnen 24 h etwa $80 \%$ der verabreichten Dosis als ${ }^{14} \mathrm{CO}_{2}$ und weniger als $1 \%$ als unverändertes 1 -Butanol abgeatmet. Etwa $1 \%$ wurde in dieser Zeit mit den Faeces ausgeschieden und maximal $5 \%$ über die Nieren mit dem Urin [19]. Der Anteil des konjugierten 1-Butanol-glucuronids bzw. -sulfats an der insgesam.t im Urin ausgeschiedenen Menge nimmt mit steigender Exposition ab und der Anteil des freien 1-Butanols zu. Bei einer Exposition gegenüber bis zu 9 mg/ $\mathrm{m}^{3}$ wurde bei beruflich exponierten Personen ausschließlich konjugiertes 1-Butanol gefunden [20].

Bundesgesundheitsbl 2014 · 57:733-743 DOI 10.1007/s00103-014-1972-x

c) Springer-Verlag Berlin Heidelberg 2014

Bekanntmachung des Umweltbundesamtes

Richtwerte für 1-Butanol in der Innenraumluft. Mitteilung der Adhoc-Arbeitsgruppe Innenraumrichtwerte der Kommission Innenraumlufthygiene und der Obersten Landesgesundheitsbehörden

\section{Zusammenfassung}

Zum Schutz der Gesundheit der Bevölkerung setzt die Ad-hoc-Arbeitsgruppe Innenraumrichtwerte der Kommission Innenraumlufthygiene und der Obersten Landesgesundheitsbehörden Richtwerte für die Innenraumluft fest. Für eine gesundheitliche Bewertung von 1-Butanol in der Luft liegen keine hinreichend aussagekräftigen Humanstudien vor. In einer gut dokumentierten und als zuverlässig eingestuften oralen Studie an Ratten wurde eine Beeinträchtigung der embryonalen Entwicklung beobachtet. Aus dieser Studie hat die US-amerikanische Umweltbehörde mittels Benchmark-Modellierung eine $\mathrm{BMDL}_{10 \text { Embryotox }}=26,1 \mathrm{mg} / \mathrm{kg} \mathrm{KG} \mathrm{Tag}$ abgeschätzt. Die Ad-hoc-Arbeitsgruppe wählt diese nachteilige Wirkungsdosis als Ausgangspunkt für die Richtwertableitung. Unter Berücksichtigung der Resorptionsrate bei inhalativer Aufnahme mit einem Faktor von 0,6, mit einer allometrischen Extrapolation von Ratte auf Mensch (Faktor 4), einem Interspeziesfaktor für Toxikodynamik von 2,5 sowie einem Faktor von 10 zur Berücksichtigung individueller Unterschiede (Intraspeziesfaktor) ergibt sich ein gerundeter Richtwert II (Gefahrenwert) von $2 \mathrm{mg} 1-\mathrm{Butanol} / \mathrm{m}^{3}$. Aus derselben Studie lässt sich eine $B M D L_{5}$ stellvertretend für eine NOAEL berechnen. Der Richtwert I (Vorsorgewert) wird ausgehend von einer $\mathrm{BMDL}_{5}$ von 12,4 mg/kg KG Tag abgeleitet. Unter Anwendung derselben Extrapolationsfaktoren ergibt sich ein Richtwert I von 0,7 mg 1-Butanol/ $\mathrm{m}^{3}$ Innenraumluft.

\section{Schlüsselwörter}

1-Butanol · Innenraumluft .

Entwicklungstoxizität · Benchmarkdosis . Richtwert

\section{Guide values for 1-butanol in indoor air. Report of the German Ad Hoc Working Group on Indoor Guidelines of the Indoor Air Hygiene Committee and of the States' Supreme Health Authorities}

\section{Abstract}

The German Ad Hoc Working Group on Indoor Guidelines of the Indoor Air Hygiene Committee and the States' Supreme Health Authorities is issuing indoor air guide values to protect public health. No human studies of sufficient quality are available for the evaluation of 1-butanol in indoor air. In a well-documented oral study on reproduction toxicity in rats, assessed as reliable, impairment of embryo development was observed. Benchmark modeling of the study data by US-EPA revealed a $\mathrm{BMDL}_{10}$ of $26.1 \mathrm{mg} / \mathrm{kg}$ b.w. per day. The working group used this $\mathrm{BMDL}_{10}$ as the point of departure for the derivation of the guide value II. Considering a human respiration rate of $20 \mathrm{~m}^{3}$ per day and a human body weight of $70 \mathrm{~kg}$, this dose was converted into an inhalative concentration. Applying a factor of 0.6 to account for the inhalative absorption rate, an allometric extrapolation factor from rat to human (factor 4), an interspecies factor of 2.5 for toxicodynamics, and a factor of 10 to account for individual differences (intraspecies factor), results in a health hazard guide value (RW II) of $2 \mathrm{mg} 1$-butanol $/ \mathrm{m}^{3}$. The benchmark dose calculation of the same study generated a BMDL 05 of $12.4 \mathrm{mg} / \mathrm{kg}$ b.w. per day. Applying the same assessment factors as for RW II, a precautionary guide value (RW I) of $0.7 \mathrm{mg} 1$-butanol $/ \mathrm{m}^{3}$ indoor air is calculated.

\section{Keywords}

1-butanol · Indoor air · Developmental toxicity - Benchmark dose - Guide value
Die Ausscheidung von 1-Butanol im Urin kann zur Beurteilung der äußeren Belastung am Arbeitsplatz herangezogen werden. Bei nicht gewerblich mit 1-Butanol belasteten Personen wurden 20-47 $\mu \mathrm{g}$ 1-Butanol/l (frei und konjugiert) im Urin gefunden (Bestimmungsgrenze $20 \mu \mathrm{g} / \mathrm{l}$ ).
Eine mittlere Konzentration von 4,5 mg 1-Butanol/ $\mathrm{m}^{3}$ Luft am Arbeitsplatz führte bei Schichtende zu einer Konzentration an freiem und konjugiertem 1-Butanol im Urin von $208 \mu \mathrm{g} / \mathrm{l}$. Für eine Belastung in Höhe des MAK-Werts von $310 \mathrm{mg} / \mathrm{m}^{3}$ wurde ein vorläufiger BAT- 
Wert von $10 \mathrm{mg}$ 1-Butanol/g Kreatinin bei Schichtende und 2 mg 1-Butanol/g Kreatinin $16 \mathrm{~h}$ nach Schichtende festgelegt (jeweils als Summe des freien und konjugierten 1-Butanols) [20,21].

\section{Wirkungen}

$\mathrm{Zu}$ gesundheitlichen Wirkungen von 1-Butanol beim Menschen liegen nur wenige Befunde vor. Berichtet wurden bei Personen mit inhalativer Exposition gegenüber 1-Butanoldämpfen am Arbeitsplatz in erster Linie Reizwirkungen und Entzündungen an den Augen, daneben wurden auch Reizungen der Schleimhäute von Nase und Rachen sowie zentralnervöse Effekte wie Kopfschmerzen und Schwindel und unerträgliche Geruchsbelästigung genannt. Auch über eine Beeinträchtigung des Hörvermögens wurde berichtet, wobei jedoch der Einfluss einer gleichzeitigen Lärmbelästigung am Arbeitsplatz schwer einzuschätzen ist. Allgemein erschweren mehrere Faktoren die Bewertung der Befunde im Hinblick auf eine Rolle von 1-Butanol als Auslöser der genannten Beschwerden: So wurde in Untersuchungen, in denen 1-Butanol eines der in erster Linie verwendeten Lösemittel darstellte, die Exposition unzureichend quantifiziert, während in anderen Untersuchungen Mischexposition mit anderen Lösemitteln vorlag und nur ein geringer Teil der Gesamtexposition gegenüber Lösemitteln auf 1-Butanol zurückzuführen war $[2,18]$.

Im Tierversuch treten bei akuter Exposition Reizwirkungen auf die Atemwege sowie zentralnervöse, sedierende Wirkungen auf. Bei fortgesetzter Exposition wurden Beeinträchtigungen der neuromuskulären Koordination, leichtere Blutbildveränderungen und in Studien zur Reproduktionstoxizität Beeinträchtigungen der Embryonalentwicklung mit vermehrtem Auftreten von Variationen und Anomalien beobachtet.

\subsection{Irritative Wirkungen}

Bei Beschäftigten, die am Arbeitsplatz 1-Butanol ausgesetzt waren, wurden in mehreren Untersuchungen Augenschäden mit Entzündungen der Hornhaut beschrieben. Dabei kam es zu Lidschwel- lungen, Rötungen, Brennen und Jucken der Augen, Fremdkörpergefühl und Vakuolenbildung im Korneaepithel. Letztere werden als Flüssigkeitseinschlüsse im Epithel angesehen, die sich infolge der Penetration von 1-Butanol in das Epithelgewebe bilden. Die genannten Wirkungen waren nach längerer Abwesenheit vom Arbeitsplatz reversibel [22, 23].

In einer über einen Zeitraum von 10 Jahren wiederholt vorgenommenen Untersuchung traten bei anfänglich durchschnittlich $606 \mathrm{mg} / \mathrm{m}^{3}$ mit Ausnahme der Vakuolenbildung die genannten Augenreizungen auf, die z. T. mit Entzündungen der Kornea, Tränenfluss, verschleiertem Sehen und erhöhter Lichtempfindlichkeit einhergingen. Nachdem die Exposition auf durchschnittlich $303 \mathrm{mg} / \mathrm{m}^{3}$ verringert worden war, wurden Augenreizungen seltener und mit Expositionsspitzen in Verbindung gebracht [24].

In einer toxikokinetischen Studie wird angeführt, dass nach 2-stündiger Exposition mit bis zu $606 \mathrm{mg} / \mathrm{m}^{3}$ 1-Butanol keiner der 12 Probanden durch die Exposition beeinträchtigt worden sei [17]. In einer Studie an 10 Probanden zur Reizwirkung verschiedener Lösemittel wurden hingegen bei kurzzeitiger Einwirkung von 1-Butanol über 3-5 min als subjektive Symptome von den Probanden bei $76 \mathrm{mg} / \mathrm{m}^{3}$ leichte Reizungen in Augen, Nase und Rachen angegeben. Bei $152 \mathrm{mg} / \mathrm{m}^{3} \mathrm{klag}$ ten die Probanden über stärkere Rachenreizung, einige auch über leichte Kopfschmerzen [25].

In einer weiteren Untersuchung speziell zum augenreizenden Potenzial von 1-Butanol wurden 8 Probanden mittels einer Augenmaske auf einem Auge gegen 1-Butanol $\left(0,300,952\right.$ bzw. $\left.3000 \mathrm{mg} / \mathrm{m}^{3}\right)$, auf dem anderen gegen Luft mit und ohne $\mathrm{CO}_{2}$-Zusatz exponiert. Neben subjektiven Angaben zur Augenreizung, die alle 5 min notiert wurden, wurden vor und nach der 60-minütigen Expositionsphase auch objektivierbare klinische Parameter erfasst. Die berichtete Intensität der $\mathrm{Au}$ genreizung wurde durch die Anwesenheit von Butanol nicht beeinflusst. Bei den beiden niedrigeren Expositionskonzentrationen war die konjunktivale Hyperämie leicht, bei der höchsten Konzentration statistisch signifikant gegenüber der
Kontrolle erhöht. Zytologische Veränderungen in der Konjunktivalflüssigkeit fanden sich nicht [26]. Nach kurzzeitiger Einwirkung über 1-3 s wurde eine Wirkungsschwelle für eine Reizung der Augen von $9000 \mathrm{mg} / \mathrm{m}^{3}$ berichtet [27].

Die Reizwirkung von 1-Butanol in der Nasenschleimhaut wurde in einer Untersuchung an Probanden anhand der Lateralisationsschwelle bestimmt. Dieser Wert bezeichnet die Konzentration, bei der Versuchspersonen in der Lage sind, bei getrennter Exposition beider Nasenlöcher anzugeben, auf welcher Seite der inhalierte Stoff einwirkt. Eine derartige, einseitig als Stechen, Brennen, Kribbeln oder auch als Kälte- oder Hitzegefühl empfundene sensorische Reizung weist darauf hin, dass die Empfindung durch den Trigeminus vermittelt wird und nicht auf reinem Geruchsempfinden über den Riechnerv beruht, da in dem Fall eine derartige Lateralisation nicht möglich ist [28]. In dem Versuch atmeten 32 Personen jeweils über beide Nasenlöcher getrennt Luft aus 2 Fläschchen ein, die gereinigtes geruchloses Mineralöl oder darin gelöstes 1-Butanol enthielten. Die Geruchschwelle, ab der ein Geruch wahrgenommen, aber nicht lateral zugeordnet werden konnte, lag im Median bei $0,48 \mathrm{mg} / \mathrm{m}^{3}$, der Mittelwert bei $9 \mathrm{mg} / \mathrm{m}^{3}$. Der Median bzw. der Mittelwert der Lateralisationsschwelle lag mit $6969 \mathrm{mg} / \mathrm{m}^{3}$ bzw. $6945 \mathrm{mg} / \mathrm{m}^{3}$ erheblich höher.

Eine Untersuchung berichtet bei einem Geruchschwellenwert von $1,2 \mathrm{mg} / \mathrm{m}^{3}$ eine verzögerte Dunkeladaptation und bei geringfügig niedrigerer $\left(0,7 \mathrm{mg} / \mathrm{m}^{3}\right)$ bzw. höherer $\left(1,5-2,5 \mathrm{mg} / \mathrm{m}^{3}\right)$ Konzentration eine verzögerte Reaktionszeit bzw. eine Hemmung des konditionierten Lidblinkreflexes [29]. Eine Bewertung und Einordnung dieser Befunde ist wegen fehlender Angabe von Details zum Studiendesign nicht möglich.

Im Tierversuch mit männlichen Ssc:CF1-Mäusen wurde die sensorische Reizwirkung von 1-Butanol anhand der Abnahme der Respirationsrate untersucht. Nach einer 1-minütigen Einwirkung verursachte eine Konzentration von $35.500 \mathrm{mg} / \mathrm{m}^{3}$ eine Verminderung der Atemrate um $50 \%\left(\mathrm{RD}_{50}\right)$. Bei fortgesetzter Exposition mit Konzentrationen unterhalb von $9100 \mathrm{mg} / \mathrm{m}^{3} \mathrm{klang}$ 
die Reaktion ab, während höhere Konzentrationen eine erneute Verminderung der Atemrate bewirkten. Dies könnte mit einer Reizung von Rezeptoren in tieferen Abschnitten des Atemtrakts in Zusammenhang stehen, auch eine beginnende narkotische Wirkung auf das zentrale Nervensystem könnte zu diesem Effekt beigetragen haben [2]. In 2 weiteren Studien an männlichen Balb/c-Mäusen war die Abnahme der Atemrate ebenfalls in der ersten Minute am stärksten, hier wurden $\mathrm{RD}_{50}$-Werte von $9100 \mathrm{mg} / \mathrm{m}^{3}$ bzw. $13.000 \mathrm{mg} / \mathrm{m}^{3}$ ermittelt $[30,31]$.

\subsection{Neurotoxizität}

Bei beruflich über längere Zeiträume gegenüber 1-Butanol exponierten Beschäftigten liegen keine bewertungsrelevanten Angaben zu neurotoxischen Wirkungen vor.

Akute zentralnervöse Wirkungen wurden in einem Verhaltenstest an Mäusen untersucht. Nach einer 4-stündigen Exposition wurde die Verkürzung der normalen anfänglichen immobilen Phase bis zum Beginn von Fluchtschwimmbewegungen in einem Wasserbecken im Vergleich zur unbehandelten Kontrollgruppe erfasst. Die Konzentration an 1-Butanol, die zu einer Abnahme der Immobilität um $50 \%$ führte, lag bei $1870 \mathrm{mg} / \mathrm{m}^{3}$ [32]. In einer anderen Studie wurde die Verminderung der Reaktion auf einen Schmerzreiz 1 min nach Ende einer 4-stündigen Exposition männlicher Ratten bzw. 2-stündiger Exposition weiblicher Mäuse gemessen. Eine um $30 \%$ reduzierte Reaktion trat bei einer 1-Butanolkonzentration von $10.000 \mathrm{mg} / \mathrm{m}^{3}$ bzw. $7300 \mathrm{mg} / \mathrm{m}^{3}$ auf [33].

In Rotarod-Tests wurde die Beeinträchtigung der neuromuskulären Koordination bei männlichen Wistar-Ratten unmittelbar nach einer 4-stündigen Exposition gegenüber 1-Butanol untersucht. In 2 Testreihen wurden $\mathrm{EC}_{50}$-Werte von $19.800 \mathrm{mg} / \mathrm{m}^{3}$ bzw. $22.920 \mathrm{mg} / \mathrm{m}^{3}$ ermittelt $[30,31]$. Als NOAEC lässt sich eine Konzentration von etwa $7575 \mathrm{mg} / \mathrm{m}^{3}$ abschätzen.

In einer subchronischen Inhalationsstudie mit männlichen Wistar-Ratten wurden je 12 Tiere/Gruppe (24 in der Kontrolle) an 6 h/Tag, 5 Tage/Woche 3 Monate gegenüber 0, 154 bzw. 308 mg
1-Butanol $/ \mathrm{m}^{3}$ exponiert [34]. Wirkungen auf die neuromuskuläre Koordination wurden mithilfe des Rotarod-Tests untersucht. Dazu wurden die Tiere vor Beginn des Versuchs 10 Tage lang trainiert, mindestens 2 min auf dem rotierenden Stab zu balancieren, und anschließend vor Beginn der Exposition und jeweils monatlich während der Studie untersucht. Bei den gegenüber 1-Butanol exponierten Tieren waren im Rotarod-Test zeit- und konzentrationsabhängig Verschlechterungen (zunehmende Zahl von Fehlversuchen) zu verzeichnen. Nach einer Auswertung der im Original als Grafik präsentierten Daten durch die US EPA [2] lag der Prozentsatz an Fehlern nach 1, 2 bzw. 3 Monaten bei der niedrigeren Konzentration bei 2, 10 bzw. $16 \%$, bei der höheren Konzentration bei 16, 25 bzw. 34\% (Kontrolle: zu allen Zeiten $0 \%$ Fehler). Die Verschlechterungen waren bei der höheren Konzentration ab dem 2. Monat signifikant.

In derselben Studie wurden auch die Wirkungen von m-Xylol (50 bzw. $100 \mathrm{ml} / \mathrm{m}^{3}$ ) und von 1:1-Gemischen aus $\mathrm{m}$-Xylol und 1-Butanol (je 50+50 oder $100+100 \mathrm{ml} / \mathrm{m}^{3}$ ) auf das Abschneiden im Rotarod-Test untersucht. Bei der niedrigeren $\mathrm{m}$-Xylolkonzentration und dem niedriger dosierten Gemisch wurden zu keinem Zeitpunkt signifikante Veränderungen gegenüber der Kontrolle beobachtet. $100 \mathrm{ml} \mathrm{m}$-Xylol/ $/ \mathrm{m}^{3}$ führten nach 1, 2 und 3 Monaten zu identischen, signifikant erhöhten Fehlerraten von jeweils ca. $35 \%$. Das Gemisch aus $100 \mathrm{ml}$ m-Xylol und $100 \mathrm{ml} 1-$ Butanol $/ \mathrm{m}^{3}$ entsprach in seiner Wirkung zu jedem untersuchten Zeitpunkt vollkommen dem von 1-Butanol allein. Die Wirkung dieses Gemisches fiel nach 1 und auch nach 2 Monaten schwächer aus als die von $100 \mathrm{ml} \mathrm{m}$ $\mathrm{Xylol} / \mathrm{m}^{3}$ allein, war nach 3 Monaten aber ebenso ausgeprägt wie durch $100 \mathrm{ml} \mathrm{m}$ $\mathrm{Xylol} / \mathrm{m}^{3}$ allein. In der Publikation [34] fehlen Angaben, wie oft die Tiere zu jedem Zeitpunkt bei jeder Konzentration den Rotarod-Test absolvieren mussten, noch wurden Streuungsmaße angegeben.

Eine Studie, in der nach Exposition von Ratten oder Mäusen über $4 \mathrm{Mo-}$ nate $\mathrm{ab} 6,6 \mathrm{mg} / \mathrm{m}^{3}$ zentralnervöse, klinisch-chemische und hormonelle Veränderungen genannt werden, ist wegen Fehlens wesentlicher Angaben nicht be- wertbar [35]. Dies gilt wegen ungenügender Darstellung von Methodik und Ergebnissen auch für die Aussagekraft einer älteren Studie an Meerschweinchen, in der nach subchronischer Exposition gegenüber $300 \mathrm{mg} / \mathrm{m}^{3}$ verminderte Erythrozyten- und Lymphozytenzahlen im Blut sowie Veränderungen der Leber und der Niere berichtet wurden [36].

Weiterhin liegen Befunde einer Inhalationsstudie an männlichen und weiblichen Sprague-Dawley-Ratten zur Neurotoxizität von 1-Butylacetat vor, das im Organismus sehr rasch unter Bildung von 1-Butanol hydrolysiert wird. In dieser Untersuchung wurden je 30 bis 40 Tiere aus beiden Geschlechtern 6 h/Tag, 5 Tage/Woche, 13 bis 14 Wochen gegenüber 0 , 2400, 7200 oder $14.000 \mathrm{mg}$ 1-Butylacetat/ $\mathrm{m}^{3}$ exponiert. Untersucht wurden das Abschneiden der Tiere in einer FOB (functional observational battery) und in operanten Verhaltenstests, ihre motorische Aktivität sowie neurohistopathologische Veränderungen. Während der Exposition mit 7200 bzw. $14.000 \mathrm{mg} / \mathrm{m}^{3}$ waren die Tiere vorübergehend sediert und vermindert aktiv. Neurotoxische Wirkungen oder histologische Veränderungen des Nervensystems traten jedoch nicht auf [37].

In einer parallel $\mathrm{zu}$ dieser Neurotoxizitätsstudie durchgeführten subchronischen Inhalationsstudie mit demselben Expositionsprotokoll traten bei der niedrigsten Konzentration keine behandlungsbedingten Effekte auf. Ab der mittleren Dosis war die Aktivität der Tiere während der Exposition vermindert, wobei dieser Effekt im Laufe der Zeit nicht stärker ausgeprägt auftrat, Körpergewicht und Futteraufnahme sowie Milz-, Leber und Nierengewicht waren vermindert, das Hodengewicht erhöht. Histologisch zeigten sich im Nasengewebe minimale bis leichte Nekrosen des olfaktorischen Epithels [38]. Da 1-Butylacetat bereits in der Nase hydrolysiert wird, werden die lokalen Schäden auf die dabei entstehende Essigsäure bzw. den pH-Abfall im Gewebe zurückgeführt. Auch die systemischen Effekte auf Organgewichte und Futteraufnahme könnten nach Einschätzung des Ausschusses für Gefahrstoffe Folge der lokalen Gewebsschädigung sein [39].

In einer nicht vollständig verfügbaren subchronischen Studie an Ratten 
(je 30 Männchen und Weibchen/Dosis) mit oraler Verabreichung von 0, 30, 125 bzw. 500 mg 1-Butanol/kg KG Tag in Wasser per Schlundsonde verursachte die höchste Dose während der letzten 6 Versuchswochen direkt nach der Verabreichung Hypoaktivität und Ataxie, die binnen $1 \mathrm{~h}$ wieder abklangen. Makroskopische oder histologische Veränderungen wurden bei Studienende nicht beobachtet. Bei einer Zwischenuntersuchung nach 6 Wochen waren einige hämatologische und klinisch-chemische Parameter in der mittleren und hohen Dosis (insbesondere Hämatokrit, Erythrozytenzahl, Hämoglobingehalt bei Weibchen vermindert) geringfügig verändert, ohne dass in jedem Fall eine Dosisabhängigkeit erkennbar gewesen wäre. Am Ende des Versuchszeitraums waren keine derartigen Veränderungen mehr nachweisbar. Die US-amerikanische Umweltbehörde geht auf Basis der vorliegenden Daten von einem NOAEL von 125 mg/kg KG Tag aus [2].

Untersuchungen mit chronischer Expositionsdauer liegen nicht vor.

\subsection{Reproduktionstoxizität}

Bewertungsrelevante Befunde beim Menschen liegen nicht vor.

In Untersuchungen an Ratten zeigten sich nach kontinuierlicher inhalativer Exposition männlicher oder weiblicher Tiere mit bis zu $18.000 \mathrm{mg}$ 1-Butanol/m während der Trächtigkeit bzw. ab Tag 6 vor der Verpaarung behandelter Männchen mit unbehandelten Weibchen keine Beeinträchtigungen der Fertilität [40]. Eine Exposition in dieser Höhe an $6 \mathrm{~h} / \mathrm{Tag}$ und mindestens 65 Tagen binnen 14 Wochen hatte außerdem keine erkennbaren toxischen Wirkungen auf die Hoden [1]. In einer weiteren Untersuchung ließen $7250 \mathrm{mg}$ 1-Butylacetat $/ \mathrm{m}^{3}$, verabreicht $\mathrm{ab}$ 3 Wochen vor der Verpaarung und während der Trächtigkeit, keinen Einfluss auf die Fertilität weiblicher Tiere erkennen [1].

Hingegen wurden in Untersuchungen an Ratten Beeinträchtigungen der Embryonalentwicklung festgestellt. Nach Exposition trächtiger Sprague-Dawley-Ratten (jeweils 15 pro Dosis) mit $0,11.000,18.000$ oder $24.000 \mathrm{mg} / \mathrm{m}^{3}$ an $7 \mathrm{~h} / \mathrm{Tag}$ vom 1 . bis 19. Tag der Gestation war das Fetalgewicht ab $18.000 \mathrm{mg} / \mathrm{m}^{3}$ erniedrigt. In allen gegenüber 1-Butanol exponierten Gruppen waren die Zahl der Würfe mit Föten, die Fehlbildungen des Skeletts (rudimentäre zervikale Rippen) aufwiesen, sowie die Zahl der davon betroffenen Föten im Vergleich zur Kontrolle signifikant erhöht. In der höchsten Dosierung traten zudem vermehrt nicht näher bezeichnete viszerale Fehlbildungen auf. Die höchste geprüfte Konzentration wirkte bereits maternal toxisch (vermindertes Körpergewicht am Ende der Gestation, erhöhte Mortalität, in einer Voruntersuchung Narkose bei der Hälfte aller Tiere) [2, 41]. Aus diesen Befunden ergibt sich nach den Auswertungen der US-amerikanische Umweltbehörde eine LOAEC (für erhöhte Inzidenz von Würfen mit Skelettfehlbildungen) von $11.000 \mathrm{mg} / \mathrm{m}^{3}$ (keine NOAEC).

In einer Studie zu Auswirkungen einer pränatalen Exposition auf das postnatale Verhalten wurden trächtige Sprague-Dawley-Ratten (15/Dosis) vom 1. bis 19. Tag der Gestation für $7 \mathrm{~h} / \mathrm{Tag}$ gegenüber 0, 9000 bzw. $18.000 \mathrm{mg} / \mathrm{m}^{3}$ exponiert. Eine Gruppe männlicher Tiere wurde 6 Wochen entsprechend exponiert und anschließend mit unbehandelten Weibchen verpaart. Unter den Nachkommen wurde ein Teil der Tiere bis maximal 60 Tage nach der Geburt ohne weitere Exposition aufgezogen und in einer Reihe von Verhaltenstests auf neuromotorische Koordination (darunter auch ein Rotarod-Test), Aktivität und Lernvermögen sowie Veränderungen in der Konzentration an Neurotransmittern im Hirngewebe hin untersucht. Hinweise auf eine Beeinträchtigung der genannten Parameter fanden sich nicht (NOAEC: $\left.18.000 \mathrm{mg} / \mathrm{m}^{3}\right)[2,40]$.

Weiterhin liegen 2 Studien zur Entwicklungstoxizität bei oraler Exposition gegenüber 1-Butanol vor. In einer der beiden Studien [42] erhielten trächtige Sprague-Dawley-Ratten (20/Dosis) bis zum 20. Tag der Gestation 1-Butanol im Trinkwasser [0, 0,2, 1 bzw. $5 \%$, entspr. 0, 316, 1454 bzw. 5654 mg/kg KG Tag]. In der höchsten Dosierung waren Futterverbrauch und Wasseraufnahme der Muttertiere sowie das Fötusgewicht um mindestens $10 \%$ gegenüber den Kontrollen reduziert. Die Länge der Föten sowie die Inzidenz externer oder viszeraler Fehlbildun- gen waren nicht erhöht. In der höchsten Dosierung traten jedoch signifikant häufiger Würfe mit Skelettvariationen (vor allem überzählige Rippen) und verzögerter Ossifikation auf. Außerdem nahm die Zahl der Würfe, in denen Tiere mit Resten an Thymusgewebe im Nackenbereich auftraten, dosisabhängig, jedoch nicht signifikant zu. Aus diesen Befunden ergibt sich eine NOAEL von $1454 \mathrm{mg} / \mathrm{kg} \mathrm{KG} \mathrm{Tag} \mathrm{und}$ eine LOAEL für entwicklungstoxische Effekte von $5654 \mathrm{mg} / \mathrm{kg} \mathrm{KG} \mathrm{Tag.}$

In der zweiten Studie [43] wurden 10 Wochen alte weibliche Wistar-Ratten (11-17/Dosis) 8 Wochen vor der Verpaarung, während der maximal 3-wöchigen Verpaarungsphase und weiter bis zur Schnittentbindung am 20. Tag der Trächtigkeit gegenüber 1-Butanol im Trinkwasser exponiert [0, 0,24, 0,8 bzw. $4 \%$, entspr. 0, 300, 1000 bzw. 5000 mg/kg KG Tag]. Wasser- und Futterverbrauch sowie Gewichtsentwicklung der Muttertiere wurden durch die Exposition nicht signifikant beeinflusst. Das Fötusgewicht wurde nicht beeinflusst, die fötale Kopf-Rumpf-Länge nahm dosisabhängig geringfügig ab (signifikant nur in der höchsten Dosierung). Der Prozentsatz von Föten bzw. Würfen mit Tieren mit verzögerter Verknöcherung des Skeletts nahm dosisabhängig zu, die Zunahme war in der höchsten Dosierung signifikant. Beobachtet wurden außerdem bei allen Dosierungen signifikant vermehrt Föten bzw. Würfe mit Föten mit morphologischen Veränderungen im Gehirn in Form eines erweiterten Subarachnoidalraums $(0,14,25,78 \%$ der Würfe) bzw. eines erweiterten dritten/erweiterter lateraler Ventrikel $(8,57$, 67 bzw. $78 \%$ der Würfe). Ab der mittleren Dosierung traten außerdem signifikant häufiger Hydrocephali auf, allerdings ohne erkennbare Dosisabhängigkeit. Aus diesen Befunden ergibt sich ein LOAEL von $300 \mathrm{mg} / \mathrm{kg}$ KG Tag (Effekt: vermehrt Würfe mit Föten mit erweitertem Subarachnoidalraum sowie der lateralen und/oder des dritten Ventrikels).

Untersuchungen mit 1-Butanol an anderen Tierarten liegen nicht vor. In einer Untersuchung mit 1-Butylacetat an Kaninchen, die während des 1 . bis 19. Tags der Trächtigkeit $7 \mathrm{~h} /$ Tag exponiert wurden, traten bei der einzigen getesteten Konzentration von 7250 mg/m³ morpho- 
logische Variationen der Gallenblase sowie unsymmetrisch verschmolzene Brustbeine und retinale Falten auf, andere Wirkungen wurden nicht beobachtet [44]. Bei Ratten waren nach Exposition mit 1-Butylacetat in der genannten Höhe bei den Föten vermehrt Rippenanomalien festzustellen, allerdings nur bei Muttertieren, die vom 7 . bis 16 . oder 1 . bis 16 . Tag der Trächtigkeit behandelt worden waren, nicht aber in einer Gruppe, in der die Muttertiere bereits 3 Wochen an $5 \mathrm{Ta}$ gen/Woche vor der Verpaarung und anschließend bis zum 16. Tag exponiert worden waren. In allen behandelten Gruppen waren Futteraufnahme und Körpergewicht der Muttertiere zum Ende der Studie ebenso vermindert wie die Länge der Föten [44].

\subsection{Kanzerogenität und Gentoxizität}

\section{Kanzerogenität}

Es liegen keine belastbaren Human- oder tierexperimentellen Studien zu diesem Endpunkt vor. Dies gilt auch für n-Butylacetat.

\section{Gentoxizität}

Bei Bakterien wurden in An- und Abwesenheit von exogenem metabolischem Aktivierungssystem keine mutagenen Wirkungen festgestellt. An Säugerzellen in vitro liegen nur Befunde von Untersuchungen ohne exogenes metabolisches Aktivierungssystem vor; dabei fanden sich keine Hinweise auf eine Induktion von Schwesterchromatidaustauschen (SCE) in CHO-Zellen oder Mikrokernen in V79-Zellen. In Kulturen von V79-Zellen traten vermehrt polyploide Zellen auf, allerdings erst bei hoher Konzentration von 0,1 mol 1-Butanol/1, die bereits erhebliche zytotoxische Wirkungen hatte. Auch die mRNA-Synthese in HeLa-Zellen wurde durch hohe Konzentrationen von 1-Butanol $(0,25 \%)$ gehemmt. Eine derartige Hemmung der mRNA-Synthese bzw. der DNA-Replikation konnte auch im zellfreien System bei 1\% 1-Butanol bzw. an bestrahlten Bakterienzellen bei 0,8\% 1-Butanol beobachtet werden [2].

Auch n-Butylacetat, das in vivo rasch enzymatisch unter Bildung von 1-Butanol hydrolysiert wird, hatte auf Bakterien keine mutagene Wirkung, verursachte in Hefezellen keine mitotischen Aneuploidien und in Lungenfibroblasten des Chinesischen Hamsters weder Chromosomenaberrationen noch Polyploidien [45].

Nach Injektion in die innere Eihülle von Hühnereiern führte 1-Butanol weder zu vermehrten SCE noch zu vermehrten Chromosomenbrüchen [2]. In der einzigen vorliegenden In-vivo-Untersuchung an Säugern zeigten sich nach oraler Verabreichung von $2000 \mathrm{mg} /(\mathrm{kg} \mathrm{KG}$ · Tag) an männliche und weibliche NMRI-Mäuse 24 bzw. 48 h später weder vermehrt klastogene Wirkungen noch Störungen der Mitose [1].

In der Gesamtbewertung lassen die Befunde kein gentoxisches Potenzial von 1-Butanol erkennen.

\subsection{Geruchswahrnehmung}

Die Wahrnehmungsschwelle stellt konventionsgemäß diejenige Konzentration dar, bei der die Hälfte der angebotenen Geruchsproben von dem Untersuchungskollektiv wahrgenommen wird. Nach der japanischen „Triangle Odor Bag Method“ wurde für 1-Butanol eine Geruchswahrnehmungsschwelle von $0,1 \mathrm{mg} / \mathrm{m}^{3}$ bestimmt [46]. Frühere in den Niederlanden durchgeführte Messungen erbrachten als Wahrnehmungsschwelle eine Konzentration von $0,08 \mathrm{mg} / \mathrm{m}^{3}$ [4]. Ältere Untersuchungen hatten eine Geruchsschwelle um $0,5 \mathrm{mg} / \mathrm{m}^{3}$ ergeben [28].

\subsection{Kombinationswirkung mit anderen Stoffen}

Von häufigen Mischexpositionen von 1-Butanol mit anderen Lösemitteln ist in der Praxis auszugehen. Untersuchungen zu Wirkungen entsprechender Gemische liegen jedoch kaum vor. In Inhalationsstudien an Ratten, die einem Gemisch von $\mathrm{m}$-Xylol und 1-Butanol ausgesetzt wurden, fanden sich Hinweise auf mögliche subadditive Wirkungen bei subchronischer Exposition [34].

Der Abbau von 1-Butanol in der Leber wird durch Ethanol kompetitiv gehemmt [2]. Bei gleichzeitiger Aufnahme von Ethanol ist daher ein verzögerter Abbau von 1-Butanol wahrscheinlich. Dies dürfte insbesondere dann zutreffen, wenn Etha- nol oral zugeführt wird, da die dabei aufgenommene Menge in aller Regel erheblich höher ist als bei Inhalation aus der Innenraumluft.

\section{Bewertung}

Insgesamt liegen nur wenige toxikologische Befunde vor, die für die gesundheitliche Bewertung einer fortgesetzten inhalativen Exposition gegenüber 1-Butanol herangezogen werden können.

Die wenigen Befunde zu toxikologischen Effekten beim Menschen weisen darauf hin, dass 76 mg 1-Butanol/ $\mathrm{m}^{3}$ bei kurzzeitiger Einwirkung über wenige Minuten mit leichten und $152 \mathrm{mg} /$ $\mathrm{m}^{3}$ mit ausgeprägteren Reizungen in $\mathrm{Au}$ gen, Nase und Rachen einhergehen können. Ab $300 \mathrm{mg} / \mathrm{m}^{3}$ wurde bei kurzzeitiger Exposition der Augen eine leichte, erst bei höherer Konzentration signifikante Hyperämie der Bindehaut berichtet. Bei beruflicher Exposition ging eine durchschnittliche Belastung in dieser Höhe mit gelegentlich auftretenden Augenreizungen einher, wobei die Effekte mit kurzzeitig höheren Expositionsspitzen in Verbindung gebracht werden; bei durchschnittlich $606 \mathrm{mg} / \mathrm{m}^{3}$ traten häufig Augenreizungen auf, z. T. mit Entzündungen der Kornea, Tränenfluss, verschleiertem Sehen und erhöhter Lichtempfindlichkeit. Erst deutlich höhere Konzentrationen von nahezu $7000 \mathrm{mg} / \mathrm{m}^{3}$ führten in einer kontrollierten Studie bei Probanden zu einer Reizung des Trigeminus im Nasenraum.

Im Tierversuch führen bei akuter Exposition erst sehr hohe Konzentrationen von über $9000 \mathrm{mg} / \mathrm{m}^{3} \mathrm{zu}$ einer Abnahme der Atemrate als Ausdruck der sensorischen Reizung. Zentralnervöse Wirkungen, die sich in verändertem Abschneiden in Verhaltenstests äußern, wurden nach akuter Exposition bereits ab Konzentrationen von $1870 \mathrm{mg} / \mathrm{m}^{3}$ beschrieben. Bei subchronischer Exposition von Ratten wurden bereits bei $308 \mathrm{mg} / \mathrm{m}^{3}$ Verschlechterungen im Rotarod-Test beschrieben, die auf Beeinträchtigungen der neuromuskulären Koordination hinweisen könnten. Allerdings weist die Studie Defizite in der Methoden- und Ergebnisdarstellung auf, und in einer anderen Studie zur Entwicklungstoxizität wurden 
nach pränataler Exposition von Ratten keine derartigen Beeinträchtigungen festgestellt. Die Befunde erscheinen daher zu wenig abgesichert und werden für die Bewertung und die Ableitung der Richtwerte hier nicht herangezogen.

In reproduktionstoxischen Studien an Ratten wurden keine Beeinträchtigungen der Fertilität, wohl aber der Embryonalentwicklung festgestellt. In einer Studie mit oraler Verabreichung von 1-Butanol traten bei einer nicht maternal toxischen Dosis von 300 mg/kg KG Tag vermehrt Würfe auf, in denen Föten eine Erweiterung des Subarachnoidalraums bzw. des dritten/der lateralen Ventrikel aufwiesen. Für beide Effekte wurde von der US EPA eine Benchmarkmodellierung vorgenommen [2]. Dabei ergab sich für das Auftreten eines erweiterten Subarachnoidalraums eine $\mathrm{BMDL}_{10}$ von $121,5 \mathrm{mg} / \mathrm{kg}$ KG Tag, für das Auftreten erweiterter dritter/lateraler Ventrikel eine $\mathrm{BMDL}_{10}$ von $26,1 \mathrm{mg} / \mathrm{kg} \mathrm{KG} \mathrm{Tag.}$

Die vorliegenden Befunde zur Gentoxizität lassen keine mutagenen oder gentoxischen Wirkungen von 1-Butanol erkennen. Untersuchungen zur kanzerogenen Wirkung von 1-Butanol liegen weder beim Menschen noch bei Versuchstieren vor.

\subsection{Bestehende Regelungen}

Die US-amerikanische Umweltbehörde regt im Entwurf (2011) ihres Berichts zu 1-Butanol eine sog. Referenzkonzentration (RfC) in Höhe von $0,06 \mathrm{mg} / \mathrm{m}^{3}$ an. Ausgangspunkt der vorgeschlagenen Ableitung sind Wirkungen auf die neuromotorische Koordination, die in einer subchronischen Studie an Ratten im Rotarod-Test beschrieben wurden [34]. Die NOAEC lag in dieser Untersuchung bei $154 \mathrm{mg} / \mathrm{m}^{3}$. Mithilfe von PBPK-Modellierungen auf Basis eines Modells von Teaguarden et al. [16] wurde eine humanäquivalente NOAEC von $59 \mathrm{mg} / \mathrm{m}^{3}$ abgeschätzt; dabei diente die arterielle Konzentration von 1-Butanol im Blut als Maß der inneren Exposition. Aus dieser NO$\mathrm{AEC}_{\text {human }}$ wurde mit einem Interspeziesfaktor von 3, einem Intraspeziesfaktor von 10, einer Zeitextrapolation (10) und einem Faktor von 3 wegen unsicherer Datenbasis die genannte RfC abgeleitet [2].
Die US-amerikanische Umweltbehörde diskutiert außerdem für die orale Aufnahme von 1-Butanol eine sog. Referenzdosis (RfD) von 0,09 mg/kg KG Tag. Ausgangspunkt sind dabei Befunde zur Entwicklungstoxizität bei Ratten, insbesondere das gehäufte Auftreten von Würfen mit Jungtieren, die erweiterte laterale oder dritte Ventrikel im Gehirn aufweisen. Mithilfe einer Benchmarkmodellierung wurde für diesen Endpunkt eine BMDL $_{10}$ von $26,1 \mathrm{mg} / \mathrm{kg}$ KG Tag abgeschätzt. Mit einem Gesamtextrapolationsfaktor von 300 (Interspeziesfaktor: 10, Intraspeziesfaktor: 10, Faktor wegen unsicherer Datenbasis: 3, Zeitextrapolation: 1, da es sich um einen entwicklungstoxischen Endpunkt handelt) wurde daraus die genannte RfD berechnet [2].

Zum Schutz vor toxischen Wirkungen von 1-Butanol in Raumschiffen wurde vom US-amerikanischen Nationalen Forschungsrat [47] 1996 eine sog. SMAC (Spacecraft Maximum Allowable Concentration) für längere Expositionszeiträume ( $\geq 180$ Tage) in Höhe von $40 \mathrm{mg} / \mathrm{m}^{3}$ (12 ppm) abgeleitet [48]. Als Basis dieser Ableitung diente eine NOAEL von 500 mg/kg KG Tag aus einer subchronischen (90-tägigen) Fütterungsstudie an Ratten, aus der mithilfe allometrischer Faktoren und einer inhalativen Absorptionsrate von $40 \%$ eine NOAEC von $250 \mathrm{ml} / \mathrm{m}^{3}$ abgeschätzt wurde. Mit einem Interspeziesextrapolationsfaktor von 10 und einem Zeitextrapolationsfaktor von 2 wurde daraus eine SMAC in Höhe des genannten Werts für eine Expositionsdauer von 180 Tagen ermittelt, die in einer neueren Bewertung (2008) auch für noch längere Zeiträume (bis 1000 Tage) festgelegt wurde [47].

In der TRGS 900 ist für Butan-1-ol ein Grenzwert von $310 \mathrm{mg} / \mathrm{m}^{3}$ am Arbeitsplatz festgelegt, mit dem Zusatz „Y“, d. h., ein Risiko der Fruchtschädigung braucht bei Einhaltung des Arbeitsplatzgrenzwertes und des biologischen Grenzwertes (BGW) nicht befürchtet zu werden [49]. Als Grundlage werden die Bewertungen der Arbeitsstoff-Kommission der Deutschen Forschungsgemeinschaft genannt. Diese sieht in erster Linie die Reizwirkung von 1-Butanol auf die Augen, die ab $606 \mathrm{mg} / \mathrm{m}^{3}$, nicht aber bei $303 \mathrm{mg} / \mathrm{m}^{3}$ auftreten, als kritisch für die Bewertung an. Ergänzend angeführt wird von der Kommission, dass in der einzigen als bewertungsrelevant angesehenen Tierstudie mit oraler Verabreichung an Ratten bei $250 \mathrm{mg} / \mathrm{kg} \mathrm{KG} \mathrm{Tag} \mathrm{außer} \mathrm{einer} \mathrm{zen-}$ tralnervösen Depression keine adversen Effekte auftraten. Nach Abschätzung der Kommission wäre für einen Beschäftigten (70 kg KG, Atemrate 10 m³/Schicht, $60 \%$ pulmonale Resorption) bei einer Exposition in Höhe des MAK-Werts während der Dauer einer Schicht mit einer Aufnahme von 25 mg 1-Butanol/kg KG Tag auszugehen. Die Befunde im Rotarod-Test aus der subchronischen Studie von Korsak et al. [34] an Ratten werden hingegen von der Kommission angezweifelt und in ihrer Bewertung nicht berücksichtigt, da von derselben Arbeitsgruppe bei akuter Exposition für Einschränkungen im Rotarod-Test eine $\mathrm{EC}_{50}$ über $22.725 \mathrm{mg} / \mathrm{m}^{3}$ ermittelt wurde und sich eine NOAEC von etwa $7575 \mathrm{mg} / \mathrm{m}^{3}$ abschätzen lasse $[18,21]$.

Im Gefahrstoffrecht der EU oder von der Internationalen Krebsforschungsbehörde liegt für 1-Butanol keine Einstufung hinsichtlich kanzerogener Wirkungen vor.

\subsection{Ableitung von Richtwerten für die Innenraumluft}

Bei inhalativer Exposition von Ratten wurde in einer subchronischen Studie eine Beeinträchtigung der neuromuskulären Koordination mit einer LOAEC von $308 \mathrm{mg} / \mathrm{m}^{3}$ beschrieben. Dieser Befund erscheint angesichts von Defiziten in der Beschreibung der Versuchsdurchführung und im Vergleich mit Befunden anderer Untersuchungen, u. a. zur Entwicklungsneurotoxizität, zu wenig abgesichert und kann daher nicht zur Ableitung der Richtwerte herangezogen werden.

Als relevant für die Ableitung der Richtwerte für die Innenraumluft wird vielmehr die Beeinträchtigung der embryonalen Entwicklung nach 1-Butanol-Exposition von Ratten angesehen. Als empfindlichster Endpunkt wird dabei die vermehrte Inzidenz von Würfen gesehen, in denen Jungtiere eine Erweiterung der lateralen oder des dritten Ventrikels im Hirn aufwiesen. Für diesen Endpunkt wurde von der US-amerikanische Umweltbehörde (2011) mittels Benchmark-Modellie- 
Tab. 2 Derivation of indoor air guide values ${ }^{a}$ : key data

\begin{tabular}{|c|c|c|c|}
\hline Substance & 1-Butanol & & \\
\hline Parameter & Value/Descriptor & Dimension & Comments \\
\hline \multicolumn{4}{|l|}{ General Information } \\
\hline CLP INDEX No & $603-004-00-6$ & & \\
\hline EC No & $200-751-6$ & & \\
\hline CAS No & $71-36-3$ & & \\
\hline CLP CMR Classification & Not classified & & \\
\hline Indoor air guide value status & Final & & \\
\hline Guide value II (RW II - Health hazard value) & 2 & $\mathrm{mg} / \mathrm{m}^{3}$ & \\
\hline Guide value I (RW I - Precautionary value) & 0.7 & $\mathrm{mg} / \mathrm{m}^{3}$ & \\
\hline Conversion factor: $1 \mathrm{ml} / \mathrm{m}^{3}=$ & 3.08 & $\mathrm{mg} / \mathrm{m}^{3}$ & \\
\hline Year & 2014 & & \\
\hline \multicolumn{4}{|l|}{ Database } \\
\hline Key study/Author(s) (Year) & Sitarek et al. (1994) & & $\begin{array}{l}\text { Sitarek K et al.: Int J Occup Med Environ } \\
\text { Health 1994:7:365-370 }\end{array}$ \\
\hline Species & Rat & & \\
\hline Route/type of study & Oral (drinking water) & & \\
\hline Study length & 14 wk & & \\
\hline Inhalative exposure duration & - & & \\
\hline Critical endpoint & Brain morphology & & Embryonal development \\
\hline Point of departure (POD) & $\mathrm{BMDL}_{10}$ & & $\mathrm{BMDL}_{05}: 12.4 \mathrm{mg} / \mathrm{kg} \mathrm{bw} \mathrm{d}^{*}$ \\
\hline POD Value & 26.1 & $\mathrm{mg} / \mathrm{kg} \mathrm{bw}^{*} \mathrm{~d}$ & USEPA 2011: EPA/635/R-11/081A \\
\hline \multicolumn{4}{|l|}{ Assessment factors } \\
\hline Dose-response assessment factor & n. a. & & \\
\hline Adjusted exposure duration factor (time scaling) & n. a. & & \\
\hline Adjusted study length factor & n. a. & & Sensitive developmental stages exposed \\
\hline Route-to-route extrapolation factor & 0.286 & $\mathrm{~m}^{3} \mathrm{~d}^{-1} / \mathrm{kg}$ & $1 /\left(70 \mathrm{~kg}^{*} 20 \mathrm{~m}^{3}\right)$ (human) \\
\hline Adjusted absorption factor (inhalation/oral) & 0.6 & & Oral $100 \%$; inhalation $60 \%$ \\
\hline \multirow[t]{2}{*}{ Interspecies factor } & 4 & & allometric \\
\hline & 2.5 & & toxicodynamic \\
\hline Intraspecies factor & 10 & & General population, kinetic + dynamic \\
\hline Sensitive population factor & n. a. & & Developmental toxicity study \\
\hline Other adjustment factors Quality of whole database & n. a. & & \\
\hline \multicolumn{4}{|l|}{ Result } \\
\hline Total assessment factor (TAF) & 17.1 & & \\
\hline POD/TAF & 1.52 & $\mathrm{mg} / \mathrm{m}^{3}$ & Calculated value; Rounded guide value II: 2 \\
\hline $\mathrm{BMDL}_{05} / \mathrm{TAF}$ & $12.4 / 17.14$ & & Calculated guide value $1 ; 0.7$ \\
\hline
\end{tabular}

rung eine $\mathrm{BMDL}_{10}$ von $26,1 \mathrm{mg} / \mathrm{kg} \mathrm{KG} \mathrm{Tag}$ berechnet, die als Ausgangspunkt zur Abschätzung des Richtwerts II herangezogen wird (• Tab. 2).

Im Hinblick auf die für die Ableitung vorzunehmende Pfad-zu-Pfad-Extrapolation ist die Bedeutung eines möglichen First-pass-Effektes bei oraler Aufnahme infolge der Metabolisierung von 1-Butanol durch Aldehyddehydrogenase und P450Monooxygenasen in der Leber zu prüfen. Dies könnte dazu führen, dass bei oraler Aufnahme die innere Belastung durch
1-Butanol geringer wäre als nach inhalativer Exposition gegenüber einer äquivalenten Dosis 1-Butanol. Die vorliegenden Befunde aus Tierversuchen liefern keine belastbaren Hinweise auf eine erhöhte Empfindlichkeit bei inhalativer im Vergleich zu oraler Exposition gegenüber 1-Butanol.

\section{Richtwert II}

Für die Ableitung von Richtwerten für Butanol in der Innenraumluft werden folgende Angaben verwendet [50]: $\mathrm{BMDL}_{10}$ : 26,1 mg/kg KG Tag
Resorptionsrate oral: 1 (100\%)

Resorptionsrate inhalativ: 0,6 (60\%)

Körpergewicht Mensch: 70 kg

Atemrate Mensch: $20 \mathrm{~m}^{3} / \mathrm{Tag}$

Allometrischer Skalierungsfaktor, Ratte: 4 (nach ECHA)

Zeitextrapolation: 1 (Studie zur Entwicklungstoxizität, die das kritische Zeitfenster abdeckt)

Interspeziesfaktor Toxikodynamik: 2,5 Intraspeziesfaktor: 10 
Faktor zum Schutz besonders empfindlicher Personengruppen („Kinderfaktor“): 1 (empfindlichste Gruppe bereits erfasst)

$$
\begin{aligned}
\text { LOAEC }_{\text {human }}= & (1 / 0,6 \times 26,1 \mathrm{mg} / \mathrm{kg} \mathrm{KG} \mathrm{d} \\
& \times 70 \mathrm{~kg} \mathrm{KG}): 20 \mathrm{~m}^{3} / \mathrm{d} \\
= & 152 \mathrm{mg} / \mathrm{m}^{3}
\end{aligned}
$$

Richtwert II $=152 \mathrm{mg} / \mathrm{m}^{3}$ :

$$
\begin{aligned}
& (4 \times 2,5 \times 10 \times 1)=1,5 \mathrm{mg} / \mathrm{m}^{3} \\
& \left(\text { gerundet: } 2 \mathrm{mg} / \mathrm{m}^{3}\right)
\end{aligned}
$$

Die Ad-hoc-Arbeitsgruppe Innenraumrichtwerte legt als Richtwert II eine Konzentration von $2 \mathrm{mg}$ 1-Butanol $/ \mathrm{m}^{3}$ fest.

Dieser Wert liegt um mehr als den Faktor 20 unter der Konzentration, bei der von Probanden bei kurzzeitiger Einwirkung erste leichte Reizwirkungen angegeben wurden. Da derartige Effekte stärker von der Höhe der Konzentration als von der kumulativen Dosis abhängen, ist davon auszugehen, dass der Richtwert II einen ausreichenden Sicherheitsabstand zu derartigen akuten Wirkungen beinhaltet. Dies gilt auch für akute zentralnervöse Wirkungen.

\section{Richtwert I}

Gemäß aktualisiertem Basisschema [50] soll der Richtwert I ausgehend von einer NOAEC oder, wenn möglich, einer $\mathrm{BMDL}_{5}$ abgeleitet werden.

Als $\mathrm{BMDL}_{05}$ ergibt sich aus derselben Studie, die zur Ableitung des Richtwerts II herangezogen wurde, eine Dosis von 12,4 mg/kg KG Tag. Mit derselben Umrechnung und denselben Extrapolationsfaktoren wie für den Richtwert II ergibt sich aus der $\mathrm{BMDL}_{05}$ ein Richtwert I von $0,7 \mathrm{mg} / \mathrm{m}^{3}$.

Die Ad-hoc-Arbeitsgruppe Innenraumrichtwerte legt als Richtwert I eine Konzentration von $0,7 \mathrm{mg}$ 1-Butanol $/ \mathrm{m}^{3}$ fest.

Der Richtwert I liegt über der Geruchswahrnehmungsschwelle von 1-Butanol, sodass eine gesonderte Bewertung der geruchlichen Wahrnehmungen von Butanol erforderlich ist.

\section{Anmerkungen}

Der Textentwurf dieser Mitteilung wurde von Dr. Jens-Uwe Voss erstellt und von der Ad-hoc-Arbeitsgruppe Innenraumrichtwerte im Februar 2014 verabschiedet. Die Literaturrecherche wurde im Oktober 2012 abgeschlossen.

\section{Literatur}

1. OECD SIDS (2001) SIDS initial assessment report for SIAM 13: n-Butyl Alcohol. Cas No.: 71-363. Screening Information Data Set (SIDS) for high production volume chemicals. UNEP, Washington DC. www.chem.unep.ch/irptc/sids/oecdsids/71363.pdf

2. U.S.EPA (2011) Toxicological review of n-Butanol (external Review Draft). September 2011. EPA/ 635/R-11/081A. U.S. Environmental Protection Agency, Washington DC. http://ofmpub.epa.gov/ eims/eimscomm.getfile?p_download_id=504786

3. U.S.National Library of Medicine (2010). n-Butyl alcohol. HSDB (Hazardous Substances Database). http://toxnet.nlm.nih.gov/. Zugegriffen: 1. Okt. 2012

4. Van Doorn R, Ruijten MW, van Harreveld T (2002) Guidance for the development of a level of odor annoyance in chemical emergency response. Version 2.1, August 29, 2002. Presented at the NAC/ AEGL-meeting September 2002, Washington DC

5. Jang YS, Lee J, Malaviya A et al (2012) Butanol production from renewable biomass: rediscovery of metabolic pathways and metabolic engineering. Biotechnol J 7:186-198

6. Hofmann H, Plieninger P (2008) Bereitstellung einer Datenbank zum Vorkommen von flüchtigen organischen Verbindungen in der Raumluft. Forschungsbericht 20561 243. Arbeitsgemeinschaft ökologischer Forschungsinstitute (AGÖF) e.V., im Auftrag des Umweltbundesamts. http://www.umweltbundesamt.de/sites/default/files/medien/publikation/long/3637.pdf

7. Eis D, Helm D, Laußmann D et al (2005) Berliner Studie zu umweltbezogenen Erkrankungen. Im Auftrag des Bundesgesundheitsministeriums, Berlin. www.apug.de/archiv/pdf/Berichtsband_Berliner-Studie.pdf

8. WHO (1987) Butanols: Four isomers -1-Butanol, 2-Butanol, tert-Butanol, Isobutanol. Environmental Health Criteria 65. International Programme on Chemical Safety (IPCS). World Health Organization, Geneva

9. Moriske HJ (2000) Vol. III-4.2: Chemische Innenraumluftverunreinigungen. In: Moriske HJ, Turowski E (Hrsg) Handbuch für Bioklima und Lufthygiene. Ecomed, Landsberg

10. Umweltbundesamt (2002) Gesundheit und Umwelthygiene. Umwelt-Survey 1990/92. www.umweltbundesamt.de/themen/gesundheit/belastungdermenschenermitteln/survey/us9092/

11. Hippelein M (2004) Background concentrations of individual and total volatile organic compounds in residential indoor air of Schleswig-Holstein, Germany. J Environ Monit 6:745-752

12. Umweltbundesamt (2008) Vergleichswerte für flüchtige organische Verbindungen (VOC und Aldehyde) in der Innenraumluft von Haushalten in Deutschland. Bundesgesundheitsbl 51:109-112

13. Ostendorp G, Riemer D, Harmel K et al (2009) Aktuelle Hintergrundwerte zur VOC-Belastung in Schulen und Kindergärten in Schleswig-Holstein. Umweltmed Forsch Prax 14:135-152
14. Neumann HD, Buxtrup M, Weber M et al (2012) Vorschlag zur Ableitung von Innenraumarbeitsplatz-Referenzwerten in Schulen. Gefahrstoffe Reinhalt Luft 72:291-297

15. von Hahn N, van Gelder R, Breuer D et al (2011) Ableitung von Innenraumarbeitsplatz-Referenzwerten. Gefahrstoffe - Reinhalt Luft 71:314-322

16. Teeguarden JG, Deisinger PJ, Poet TS et al (2005) Derivation of a human equivalent concentration for $n$-butanol using a physiologically based pharmacokinetic model for n-butyl acetate and metabolites n-butanol and n-butyric acid. Toxicol Sci 85:429-446

17. Astrand I, Ovrum P, Lindqvist T et al (1976) Exposure to butyl alcohol: uptake and distribution in man. Scand J Work Environ Health 2:165-175

18. DFG (1999) 1-Butanol. In: Greim H (Hrsg) Gesundheitsschädliche Arbeitsstoffe. Toxikologischarbeitsmedizinische Begründungen von MAKWerten, 28. Lieferung:1-16. Wiley-VCH, Weinheim

19. DiVincenzo GD, Hamilton ML (1979) Fate of $n$-butanol in rats after oral administration and its uptake by dogs after inhalation or skin application. Toxicol Appl Pharmacol 48:317-325

20. DFG (2001) 1-Butanol. In: Greim H (Hrsg) Gesundheitsschädliche Arbeitsstoffe. Grenzwerte in biologischem Material, 10. Lieferung Band 1:1-7. WileyVCH, Weinheim

21. DFG (2010) 1-Butanol (n-Butyl alcohol). In: Greim $\mathrm{H}$ (Hrsg) The MAK Collection Part IV: BAT Value Documentations 5:29-33. Wiley-VCH, Weinheim

22. Cogan DG, Grant WM (1945) An unsual type of keratitis associated with exposure to n-butyl alcohol (butanol). Arch Ophthal 33:106-108 (zitiert nach [18])

23. Tabershaw IR, Fahy JP, Skinner JB (1944) Industrial exposure to butanol. J Ind Hyg Toxicol 26: 328-330 (zitiert nach [18])

24. Sterner JH, Crouch HC, Brockmyre HF et al (1949) A ten-year study of butyl alcohol exposure. Am Ind Hyg Assoc 10:53-59 (zitiert nach [2] und [18])

25. Nelson KW, Ege JF, Ross M et al (1943) Sensory response to certan industrial solvent vapors. J Ind Hyg Toxicol 25:282-285

26. Hempel-Jorgensen A, Kjaergaard SK, Molhave L (1998) Cytological changes and conjunctival hyperemia in relation to sensory eye irritation. Int Arch Occup Environ Health 71:225-235

27. Cometto-Muniz JE, Cain WS (1995) Relative sensitivity of the ocular trigeminal, nasal trigeminal and olfactory systems to airborne chemicals. Chem Senses 20:191-198

28. Wysocki CJ, Dalton P, Brody MJ et al (1997) Acetone odor and irritation thresholds obtained from acetone-exposed factory workers and from control (occupationally unexposed) subjects. Am Ind Hyg Assoc J 58:704-712

29. Baikov BK, Khachaturian MK (1973) Hygienic evaluation of the reflex action on the body of low concentrations of butyl alcohol entering the atmosphere [In russischer Sprache]. Gig Sanit 38:7-11 (zitiert nach [2])

30. Korsak Z, Rydzynski K (1994) Effects of acute combined inhalation exposure to $n$-butyl alcohol and n-butyl acetate in experimental animals. Int J Occup Med Environ Health 7:273-280

31. Korsak Z, Swiercz R, Jedrychowski R (1993) Effects of acute combined exposure to N-butyl alcohol and M-xylene. Pol J Occup Med Environ Health 6:35-41 
32. de Ceaurriz J, Desiles, Bonnet P et al (1983) Concentration-dependent behavioral changes in $\mathrm{mi}$ ce following short-term inhalation exposure to various industrial solvents. Toxicol Appl Pharmacol 67:383-389

33. Frantik E, Hornychova M, Horvath M (1994) Relative acute neurotoxicity of solvents: isoeffective air concentrations of 48 compounds evaluated in rats and mice. Environ Res 66:173-185

34. Korsak Z, Wisniewska-Knypl J, Swiercz R (1994) Toxic effects of subchronic combined exposure to n-butyl alcohol and m-xylene in rats. Int J Occup Med Environ Health 7:155-166

35. Rumyanstev AP, Lobanova IY, Tiunovy LV et al (1979) Toxicology of butyl alcohol (in russischer Sprache). Khimich promyshl ser toksikol sanit khimia plastmass 2:24-26 (Zitiert nach [2])

36. Smyth HF, Smyth HF Jr (1928) Inhalation experiments with certain laquer solvents. J Ind Hyg 10:261-271 (Zitiert nach [2])

37. David RM, Tyler TR, Ouellette R et al (1998) Evaluation of subchronic neurotoxicity of n-butyl acetate vapor. Neurotoxicol 19:809-822

38. David RM, Tyler TR, Ouellette R et al (2001) Evaluation of subchronic toxicity of n-butyl acetate vapor. Food Chem Toxicol 39:877-886

39. AGS (2012). Begründungen zu Arbeitsplatzgrenzwerten: Butylacetate. In: Ausschuss für Gefahrstoffe (Hrsg) Bundesanstalt für Arbeitsschutz und Arbeitsmedizin, Dortmund. http://www.baua.de/ de/Themen-von-A-Z/Gefahrstoffe/TRGS/pdf/900/ 900-butylacetate.pdf

40. Nelson BK, Brightwell WS, Robertson SK et al (1989) Behavioral teratology investigation of 1-butanol in rats. Neurotoxicol Teratol 11:313-315

41. Nelson BK, Brightwell WS, Khan A et al (1989) Lack of selective developmental toxicity of three butanol isomers administered by inhalation to rats. Fundam Appl Toxicol 12:469-479

42. Ema M, Hara H, Matsumoto M et al (2005). Evaluation of developmental toxicity of 1-butanol given to rats in drinking water throughout pregnancy. Food Chem Toxicol 43:325-331

43. Sitarek K, Berlinska B, Baranski B (1994) Assessment of the effect of $n$-butanol given to female rats in drinking water on fertility and prenatal development of their offspring. Int J Occup Med Environ Health 7:365-370

44. DFG (2003) n-Butyl acetate. In: Greim H (Hrsg) The MAK collection for occupational health and safety 19:79-88. Wiley-VCH, Weinheim

45. WHO (2005) Butyl Acetates. Concise International Chemical Assessment Document 64. WHO Press, Geneva

46. Nagata Y (2003) Measurement of odor threshold by triangle odor bag method. Odor Measurement Review. Japan Ministry of the Environment. http:// www.env.go.jp/en/air/odor/measure/02_3_2.pdf

47. NRC (2008) Butanol. In: National Research Council, Committee on Toxicology (Hrsg) Spacecraft Maximum Allowable Concentrations for Selected Airborne Chemicals 5:73-84. National Academy of Science. National Academy Press, Washington DC

48. NRC (1996) Butanol. In: National Research Council, Committee on Toxicology (Hrsg) Spacecraft Maximum Allowable Concentrations for Selected Airborne Chemicals 3:53-77. National Academy of Science, National Academy Press, Washington DC

49. AGS (2012) Technische Regel für Gefahrstoffe 900. Arbeitsplatzgrenzwerte (TRGS 900). Ausgabe: Januar 2006, zuletzt geändert und ergänzt: GMBI 2012:715-716 vom 13.9.2012 [Nr. 40]
50. Ad-hoc-Arbeitsgruppe Innenraumrichtwerte der IRK/AOLG (2012) Richtwerte für die Innenraumluft: erste Fortschreibung des Basisschemas. Bundesgesundheitsbl 55:279-290 